\title{
Avaliação e reabilitação vestibular no indivíduo idoso
}

Vestibular assessment and rehabilitation in the elderly

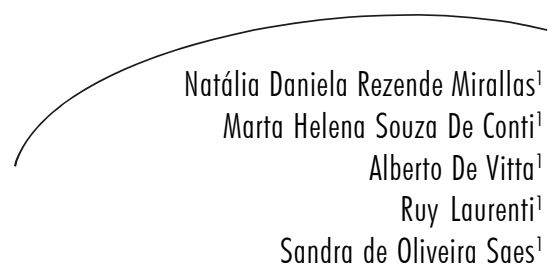

Resumo

Considerando a necessidade de propiciar melhora na qualidade de vida do idoso e a elevada incidência de queixas relacionadas aos transtornos de equilíbrio nessa faixa etária, este estudo teve como objetivo avaliar o equilíbrio estático e dinâmico de indivíduos idosos com queixas de tonturas e verificar a eficácia da reabilitação vestibular $(\mathrm{RV})$ individualizada, por meio da comparação do escore do Dizziness Handicap Inventory - DHI (questionário de handicap), antes do início da RV e no momento da alta ou, no máximo, após 12 sessões de reabilitação. Participaram da pesquisa 11 indivíduos de faixa etária acima ou igual a 60 anos, submetidos a diagnóstico otoneurológico, composto de avaliação otorrinolaringológica, audiológica, vectoeletronistagmografia e exames complementares, quando necessário. As principais queixas foram tonturas, desequilíbrio e quedas. Todos os pacientes tiveram diagnóstico de síndrome vestibular periférica, sendo dez irritativos e um deficitário unilateral. Pode-se observar que a maioria dos casos apresentou desaparecimento de seus sintomas ou significativa diminuição em suas manifestações, salvo dois pacientes que apresentavam doenças associadas ou não seguiram as orientações dadas. A RV individualizada mostrou ser um procedimento terapêutico eficaz na terceira idade e o DHI um instrumento importante no acompanhamento da evolução do paciente.

\section{Abstract}

Considering the need to improve elderly's quality of life the high incidence of complaints related to equilibrium disturbance in this age group, this study aimed to evaluate the static and dynamic equilibrium of elderly who complain of dizziness and verify the individualized vestibular rehabilitation, comparing dizziness handicap inventory - DHI (handicap questionnaire) score before starting

\footnotetext{
Programa de Pós-graduação em Saúde Coletiva. Universidade do Sagrado Coração, Bauru, SP, Brasil.
}

Correspondênca / Correspondence

E-mail: sandrasaes@gmail.com; sandra.saes@usc.br

Palavras-chave:

Envelhecimento. Labirinto. Reabilitação. Qualidade de Vida, Doença do nervo vestibular. 
to exercise and at the moment of discharge or after 12 rehabilitation sessions. Eleven individuals aged sixty years or more participated in this research, undergoing otoneurologic diagnostic, composed by otorrinilaringologic assessment, audiologic, electron vector tomography an extras exams if needed. The main complaints were dizziness, lack of equilibrium and droppings. All patients were diagnosed with peripheral vestibular syndrome, being ten irritative and one one-sided. It can be noticed that in most cases the symptoms disappeared or decreased. Except for two patients who presented diseases associated or had not followed the orientations. The individualized vestibular rehabilitation has proved to be an effective therapeutic procedure in elderly and DHI an important tool in following patient's evolution.
Key words: Aging. Labyrinth. Rehabilitation. Quality of Life. Vestibulocochlear Nerve Diseases.

\section{INTRODUÇÃO}

A população idosa vem crescendo ao longo das últimas décadas, principalmente pela redução $\mathrm{da}$ mortalidade em crianças, jovens e adultos, e também devido ao aumento da expectativa de vida. Outra razão são os avanços das ciências da saúde, que permitem a manutenção de diversas funções orgânicas e a restauração de grande parte das funções que já não ocorrem de forma adequada. Considerando o fato de ser comum o aparecimento de limitações físicas associadas a esta fase da vida, a perda da autonomia é a maior preocupação dos idosos. ${ }^{1-3}$ Consequentemente, surge a necessidade de políticas adequadas para promover melhor qualidade de vida a essa faixa etária.

Envelhecer não necessariamente significa o acúmulo de fatores de risco de doenças nãotransmissíveis. A possibilidade de envelhecer de forma saudável deveria se converter em prioridade das políticas públicas, não só pela questão da saúde, mas também pelo aspecto econômico - os idosos com incapacidades não são economicamente ativos e representam ônus para as finanças públicas. $^{4}$

O envelhecimento pode comprometer a habilidade do sistema nervoso central em processar os sinais vestibulares, visuais e proprioceptivos responsáveis pela manutenção do equilíbrio corporal, diminuindo a capacidade de modificações dos reflexos adaptativos. Tal processo degenerativo é responsável pela ocorrência de vertigem e/ou tontura e de desequilíbrio na população geriátrica, sendo este um dos principais fatores que limitam a vida do indivíduo idoso. ${ }^{5}$

O equilíbrio depende dos sistemas visual, locomotor e vestibular, e da integração de suas atividades no sistema nervoso central. A crise vertiginosa no idoso costuma ser intensa e duradoura, acompanhada de palidez, sudorese, náusea, vômito e taquicardia, às vezes por vários dias. ${ }^{6}$

Um dos principais fatores que limitam a vida do indivíduo idoso é o desequilíbrio corporal, que em aproximadamente $80 \%$ dos casos não pode ser atribuído a uma causa específica, mas ao comprometimento do sistema de equilíbrio como um todo. Em mais da metade dos casos, o desequilíbrio tem origem entre os 65 e 75 anos, e cerca de $30 \%$ dos idosos apresentam os primeiros sintomas entre os 75 e os 85 anos. Apenas 15\% dos casos apresentam algum diagnóstico a que se possa atribuir a instabilidade, permanecendo os outros $85 \%$ sem causa aparente para a situação de desequilíbrio.?

O tratamento dos idosos que apresentam limitações de locomoção minimiza o número de acidentes como quedas, favorecendo a sensação de independência e de orientação espacial. ${ }^{8}$ Para as vestibulopatias, existem opções terapêuticas como o uso de medicação antivertiginosa, tratamento cirúrgico, orientação nutricional, mudança de hábitos inadequados, psicoterapia e reabilitação vestibular (RV). ${ }^{9}$

A melhor atitude nos transtornos labirínticos é proceder por meio da múltipla abordagem 
terapêutica baseada nos achados da avaliação vestibular e no diagnóstico sindrômico, topográfico e etiológico de cada caso, apropriando-se de todos os recursos disponíveis e cabíveis em cada situação.

A identificação precisa da causa do desequilíbrio deve envolver uma avaliação clínica direcionada à queixa do paciente, doenças associadas, bem como uma avaliação integral dos sistemas envolvidos no equilíbrio corporal e suas eventuais limitações. ${ }^{10} \mathrm{~A}$ associação de alterações em múltiplos órgãos e sistemas (cardiovascular, metabólico, neurológico, sensoriais, psicológico, hormonal) como desencadeante da tontura é a grande chave para a adequada abordagem do desequilíbrio no idoso. Há necessidade de abordagem multidisciplinar (otorrinolaringologistas, geriatras, cardiologistas, psicólogos, fonoaudiólogos, fisioterapeutas) para se obter a completa reabilitação do equilíbrio nesses pacientes, minimizando assim os riscos e morbidades associadas às quedas e ao isolamento social do indivíduo. ${ }^{10}$

Nos últimos anos, cada vez mais a RV vem sendo utilizada para a melhora do quadro de desequilíbrio. A RV tem como benefício, além da diminuição dos sintomas referidos pelos portadores de labirintopatias, a melhora do convívio social do idoso. ${ }^{11}$ Trata-se de um recurso terapêutico aplicado como tratamento em pacientes com distúrbios do equilíbrio corporal, sendo a proposta de atuação baseada nos mecanismos relacionados à plasticidade neuronal do sistema nervoso central, para promover a estabilização visual durante os movimentos da cabeça, melhorar a interação vestíbulo-visual durante a movimentação cefálica, ampliar a estabilidade postural estática e dinâmica nas condições que produzem informações sensoriais conflitantes e diminuir a sensibilidade individual à movimentação cefálica. ${ }^{12}$

A RV pretende restaurar a função de equilíbrio ou trazê-la o mais próximo possível do normal, sem a terapia medicamentosa. ${ }^{13}$ Tem como objetivo a melhora do equilíbrio global, da qualidade de vida e a restauração da orientação espacial por meio de exercícios que estimulam os fenômenos de adaptação envolvendo a habituação e a compensação.

Um estudo mostrou que a intervenção terapêutica, por meio de exercícios de reabilitação, pode ser favoravelmente adotada, frente às queixas de alterações do equilíbrio corporal de idosos portadores de disfunções vestibulares periféricas de causa idiopática, aumentando a qualidade de vida dos sujeitos. ${ }^{14}$

Como medida quantitativa da evolução desses pacientes, Jacobson, em 1990,15 elaborou um questionário de handicap para tontura, o qual foi adaptado culturalmente à população brasileira por Castro em 2003, ${ }^{16}$ com o objetivo de verificar o grau de desvantagem que a disfunção vestibular causa na vida do paciente. O questionário avalia três escalas: a emocional e a funcional, com nove questões cada, e a escala orgânico-física, com sete questões, totalizando 25 questões. As respostas estipuladas e pontuadas de tal forma: "sim", equivalendo a 4 pontos, "às vezes", 2 pontos, e "não", a nenhum ponto. A pontuação total varia de $\mathrm{O}$ a 100 pontos, e quanto mais próximo de 100 , maior será a desvantagem causada pela alteração vestibular. A comparação do questionário pré e pós-reabilitação é feita por meio da avaliação da pontuação, e quando a diferença entre os resultados se encontra maior ou igual a 18 pontos, considerase efetivo o procedimento. ${ }^{14}$

Considerando a necessidade e importância de estudos e programas que proporcionem melhoria da qualidade de vida do idoso, o presente estudo visa a avaliar o equilíbrio estático e dinâmico de indivíduos idosos com queixas de tonturas e verificar a eficácia da RV individualizada, por meio da comparação do escore do Dizziness Handicap Inventory - DHI (questionário de handicap), antes do início da RV e no momento da alta ou, no máximo, após 12 sessões de reabilitação.

\section{METODOLOGIA}

O estudo foi desenvolvido na Clínica de Educação para Saúde (CEPS) da Universidade do Sagrado Coração (USC), em Bauru-SP, e o projeto 
foi aprovado pelo Comitê de Ética em Pesquisa da referida Universidade, sob protocolo de número 78/06. Foram acompanhados 11 idosos, de ambos os sexos, com idade igual ou superior a 60 anos, com diagnóstico clínico de labirintopatia periférica realizado pelo otorrinolaringologista, que encaminhou os participantes para RV. Foram excluídos indivíduos com disfunções na coluna cervical, alterações reumatológicas e/ou ortopédicas gerais e cardíacas, que impossibilitavam a realização da RV.

Os pacientes que apresentavam os critérios de elegibilidade foram encaminhados pelos otorrinolaringologistas da CEPS, para a RV. Antes de iniciarem a $R V$ propriamente dita, os pacientes foram orientados quanto aos objetivos e procedimentos do estudo, bem como sobre os princípios da RV. Todos os participantes concordaram com e assinaram o Termo de Consentimento Livre e Esclarecido.

Para sistematização diagnóstica, todos os pacientes incluídos passaram pelos mesmos procedimentos: avaliação e diagnóstico otorrinolaringológico composto por anamnese, exame clínico e encaminhamento para avaliação funcional do sistema auditivo periférico e sistema vestibular, que compreendeu a audiometria tonal limiar, logoaudiometria, imitanciometria, pesquisa do nistagmo de posição e posicional e vectoeletronistagmografia. A classificação do grau de perda auditiva foi realizada segundo o BIAP (Bureau International d'AudioPhologia) ${ }^{17}$. Os parâmetros de realização e análise da logoaudiometria e imitanciometria foram baseados em Santos \& Russo. ${ }^{18}$

Para a realização do exame vestibular, foi recomendada a abstenção de ingestão de café, chá mate e chá preto, bebida alcoólica, chocolate, cigarro, refrigerante com cafeína, nas 72 horas que antecederam a realização do exame. $O$ paciente foi orientado a não ingerir medicamentos sem a orientação médica.

A ocorrência e características do nistagmo de posicionamento e do nistagmo de posição, como latência, fatigabilidade, duração e sintomas associados foram analisadas de acordo com Herdmann ${ }^{19} \mathrm{e}$ Caovilla et al. ${ }^{20} \mathrm{~A}$ sequência e análise das demais provas da avaliação do sistema vestibular por meio da vectoelectronistagmografia foram realizadas de acordo com os critérios de Mangabeira et al. ${ }^{21}$ e Caovilla et al. ${ }^{20}$

Antes de iniciarem o processo de RV, todos os pacientes foram orientados quanto aos objetivos, aplicabilidade, fatores favoráveis e desfavoráveis, além da necessidade de colaboração e participação ativa dos mesmos. Aplicou-se então o DHI como procedimento pré-reabilitação e repetido após o término da terapia.

Iniciando a reabilitação propriamente dita, foram realizadas provas terapêuticas, a fim de instituir o planejamento terapêutico personalizado. Os exercícios prescritos tiveram como objetivo a estimulação do equilíbrio estático e dinâmico, além dos reflexos cérvico-ocular, vestíbulo-cervicais e vestíbulo-oculares. Foram indicados os exercícios que proporcionavam o aparecimento de sintomas labirínticos, sendo que os mesmos deveriam ser praticados duas vezes ao dia, durante todos os dias. O tempo de RV variou de acordo com a evolução do paciente, tendo como tempo mínimo de um mês e finalização até que os sintomas desaparecessem completamente ou não interferissem mais na rotina do paciente, ou seja, que não houvesse mais queixas.

Ressalta-se que a proposta de reabilitação labiríntica no presente estudo teve caráter individualizado, sendo portanto elaborada de acordo com o diagnóstico, sintomatologia e resultados obtidos no momento da prova terapêutica. A seguir encontram-se descritos alguns dos exercícios propostos aos pacientes.

1. deitar em decúbito dorsal e virar para decúbito ventral, fazendo o movimento dos exercícios descrito acima, elevando os braços;

2. sentar com os membros inferiores estendidos, mover para decúbito dorsal e depois para sentado novamente; 
3. sentar na cama ou no leito, com os membros inferiores para fora, levar o corpo para trás e depois para frente;

4. sentar em uma cadeira e fazer uma retroversão e antiversão com a pelve;

5. deitar em decúbito dorsal e após sentar-se, fazendo dissociação de cinturas;

6. a partir da posição sentada, ficar em pé, sem usar os membros superiores;

7. andar em linha reta olhando alternadamente para os lados;

8. andar para trás, ajustando o corpo;

9. andar para os lados, cruzando um membro inferior na frente do outro, ajustando o corpo;

10. andar reduzindo a largura dos passos, ajustando o corpo;

11. caminhar para frente e para trás realizando os movimentos de rotação e flexo-extensão de cabeça.

Para os pacientes que tiveram diagnóstico de vertigem postural, foi executada a manobra de Epley. ${ }^{13}$

Após a coleta dos dados, os resultados foram descritos individualmente e comparadas as respostas do DHI, sendo considerada como melhora a diminuição em 18 ou mais pontos na soma das escalas.

\section{RESULTADOS}

A análise dos dados revelou que, dos 11 casos estudados, oito eram do sexo feminino. As queixas mais comuns trazidas pelos participantes foram de tontura, vertigem postural, queda e náusea. As doenças mais encontradas foram depressão, hipertensão arterial, transtorno vascular, alterações de hormônios femininos e tireoideanos.

Quanto à avaliação audiológica, nove participantes apresentavam curva audiométrica descendente do tipo sensorioneural, variando o grau de leve a severo, e dois participantes apresentaram avaliação audiológica dentro dos padrões de normalidade. Todos os pacientes atendidos realizaram provas posturais e a vectoeletronistagmografia, as quais demonstraram ser importante método no diagnóstico e orientação terapêutica. Na existência de doenças associadas ou sinais sugestivos de outras morbidades, foi necessária a complementação por meio de exames laboratoriais e/ou de imagem.

Dos resultados obtidos na avaliação vestibular, oito participantes tiveram diagnóstico de síndrome vestibular periférica irritativa, e destes, cinco foram diagnosticadas pelo ORL como vertigem postural paroxística benigna (VPPB); um, deficitária unilateral; um, deficitária unilateral associada a um quadro irritativo (contra-lateral, por compensação), e um, inconclusiva. A história clínica dos pacientes apresentados foi sempre condizente com o quadro diagnosticado, ou seja, nos casos de VPPB, a presença da tontura era desencadeada por movimentos específicos do corpo e/ou cabeça.

Nos casos estudados, uma paciente se automedicava e permaneceu com esse hábito, mesmo após as orientações realizadas, ao longo de todo processo terapêutico. As orientações enfocavam os possíveis efeitos deletérios, restrições e contraindicações. Dos participantes, dois realizavam acompanhamento psicológico, pois apresentavam o quadro de depressão associado. Quanto à realização de atividade física, verificou-se que cinco não praticavam, três realizavam esporadicamente e apenas três realizavam antes de iniciarem o tratamento. Todos foram orientados quanto à importância de tal prática.

Quanto à alimentação, a maioria controlava a ingestão de gorduras e açúcares, porém quanto à regularidade de intervalo entre as refeições, seis pacientes alimentavam-se de maneira adequada, três deles de maneira regular e dois alimentavamse de maneira incorreta, realizando intervalos longos entre as refeições. Também foram orientados antes e durante todo o processo do tratamento para se alimentarem de maneira 
saudável e em horários apropriados, revelando sempre a importância desses hábitos para a melhora do quadro labiríntico.

Alguns pacientes seguiram as orientações sobre atividade física, cuidados em relação à dieta alimentar e hábitos inadequados (sedentarismo, tabagismo, consumo de álcool e automedicação). Os pacientes que adotaram essas orientações e realizaram corretamente os exercícios de RV propostos apresentaram melhora de seus sintomas. Porém, alguns que continuaram com seus hábitos inadequados, mesmo realizando os exercícios de $\mathrm{RV}$, relatavam piora na manifestação de seus sintomas após, por exemplo, fumar ou consumir bebidas alcoólicas.

Dos indivíduos atendidos, nove já haviam sido submetidos a tratamento medicamentoso, devido aos sintomas apresentados, mas não relatavam melhora; e apenas dois estavam buscando tratamento pela primeira vez e não haviam feito uso de medicamento para o quadro labiríntico.

Pode-se constatar que a múltipla abordagem diagnóstica possibilitou a escolha de condutas apropriadas para cada caso. Dos pacientes com diagnóstico de VPPB, três realizaram, como única opção terapêutica, a manobra de reposicionamento com boa resposta e alta da $\mathrm{RV}$, apresentando $\mathrm{DHI}$ pós-reabilitação com valor 0 (zero). Outros dois referiram melhora após a realização da manobra de reposição, mas por não apresentarem desaparecimento total dos sintomas, foram submetidos a exercícios personalizados de RV. Dos oito casos submetidos à RV por meio de exercícios personalizados, observou-se melhora significativa dos sintomas por meio da comparação do DHI pré e pós-reabilitação em seis pacientes, ou seja, diminuição de 18 pontos ou mais no escore do $\mathrm{DHI}$.
A aceitação e participação ativa na terapêutica da RV foram comuns entre os pacientes participantes, os quais realizavam regularmente os exercícios propostos. Contudo, a mudança de hábitos alimentares, a prática de atividades físicas e o abandono de hábitos deletérios nem sempre foram seguidos. Observou-se que dos dois casos que não apresentaram boa evolução, um manteve hábitos deletérios (tabagismo e etilismo) e um apresentava diagnóstico de depressão e fazia uso de medicação depressora do labirinto, o que possivelmente contribuiu para o insucesso terapêutico.

Neste estudo, observou-se que os pacientes submetidos a RV tiveram seus sintomas eliminados ou diminuídos, e mesmo com fatores desfavoráveis (hábitos inadequados, multimedicação, etc.), a RV monstrou-se um procedimento terapêutico que proporciona melhora sintomatológica em curto período de tratamento para os idosos. No entanto, a adesão a todas as orientações e práticas propostas para a RV é essencial para garantir bom prognóstico, uma vez que os indivíduos que não seguiram as orientações dadas apresentaram pouca ou nenhuma melhora em seus quadros.

Os resultados do $\mathrm{DHI}$, pré e pós-reabilitação vestibular, revelaram significativa melhora em todas as escalas avaliadas. A média dos resultados do DHI pré foi 42,54 pontos e do pós, 24,18 pontos, caracterizando uma melhora média de 18,36 pontos, conforme ilustrados na tabela 1 . Dos participantes, nove apresentaram melhora significativa entre o pré e o pós-reabilitação; destes, três referiram desaparecimento total dos sintomas, diminuindo a pontuação para zero. Um paciente apresentou piora em suas respostas no questionário pós-reabilitação e outro se manteve igual nos questionários pré e pósreabilitação. 
Tabela 1 - Total dos valores individuais do DHI, pré e pós-reabilitação vestibular. Baurú, SP, 2010.

\begin{tabular}{lccc}
\hline & & \multicolumn{2}{c}{ Pontuação DHI } \\
\cline { 2 - 3 } & Pacientes & Pré-reabilitação & Pós-reabilitação \\
& 1 & 34 & 00 \\
& 2 & 32 & 12 \\
& 56 & 46 & 34 \\
& 4 & 42 & 46 \\
& 5 & 64 & 44 \\
& 6 & 42 & 36 \\
& 7 & 60 & 50 \\
& 8 & 16 & 38 \\
& 9 & 48 & 06 \\
& 10 & 28 & 00 \\
& 11 & 468 & 00 \\
\hline Total & & 42,54 & 266 \\
\hline Média & & & 24,18 \\
\hline
\end{tabular}

$\mathrm{Na}$ tabela 2, encontram-se os resultados referentes à média de pontuação obtida em cada questão do DHI, pré e pós-reabilitação. Os resultados referentes à média de cada escala do DHI pré e pós-reabilitação encontram-se na tabela 3. Das três escalas analisadas pelo DHI, observouse que a escala funcional foi a que apresentou maior melhora na comparação pré e pós, seguida das escalas emocional e orgânico-física.

Como se observou neste estudo, a escala orgânico-física foi a mais afetada, provavelmente pelo fato de que os participantes apresentavam queixas posturais, evidenciadas pela escala orgânica. A maioria dos idosos apresentou melhora total ou parcial de seus sintomas porque seguiram as orientações dadas ao longo das sessões, modificaram hábitos que prejudicavam sua qualidade de vida, além de realizarem os exercícios em casa conforme orientação.

\section{DISCUSSÃO}

A diminuição dos sintomas associados à labirintopatia favorecendo uma melhora na qualidade de vida e na socialização do idoso é tema de interesse dos profissionais que atuam neste grupo etário. No presente estudo, observouse prevalência de doenças labirínticas para mulheres (8:3). Dados semelhantes são descritos na literatura, que atribui tal prevalência às alterações hormonais e metabólicas que as atingem em maior proporção e comprometem diretamente o funcionamento do sistema vestibular. ${ }^{22-24}$

Os sintomas caracterizados por tontura, vertigem postural, queda e náusea foram os mais referidos pelos participantes e comprometem significativamente a vida do indivíduo, levandoo muitas vezes a abandonar atividades ocupacionais, criar dependência de familiares e até se isolar socialmente. Estudos apontam que as principais queixas relatadas pelos pacientes idosos são transtornos do equilíbrio, zumbido, dificuldade auditiva, quedas e náusea, sintomas decorrentes de comprometimento do sistema auditivo e vestibular. ${ }^{5,25}$ Indivíduos com queixas de tonturas frequentemente relatam desconforto, embaraço social e medo da incapacidade física, e pessoas sem disfunções psiquiátricas podem desenvolver sinais de ansiedade e pânicos depois de uma crise vestibular. ${ }^{26}$

Quanto às doenças presentes, buscou-se conscientizar os pacientes das implicações destas na sintomatologia vestibular e alertá-los para a necessidade de controle sistemático dessas morbidades, conduta que colabora com a recuperação e/ou manutenção do equilíbrio. Os 
Tabela 2 - Média de pontuação obtida em cada questão do DHI, pré e pós-reabilitação. Baurú, SP, 2010.

\begin{tabular}{|c|c|c|}
\hline Questões & Pré-reabilitação & Pós-reabilitação \\
\hline O-1. Olhar para cima aumenta seu problema? & 2,91 & 1,64 \\
\hline $\begin{array}{l}\text { E-2. Sente-se frustrado(a) em razão de seu } \\
\text { problema? }\end{array}$ & 2,73 & 1,45 \\
\hline $\begin{array}{l}\text { F-3. Por causa do seu problema, você restringe } \\
\text { as viagens de trabalho ou recreação? }\end{array}$ & 2,00 & 0,73 \\
\hline $\begin{array}{l}\text { F-4. Andar no corredor de um supermercado } \\
\text { aumenta seu problema? }\end{array}$ & 1,45 & 1,09 \\
\hline $\begin{array}{l}\text { F-5. Em decorrência do seu problema, você tem } \\
\text { dificuldade para deitar-se ou levantar-se da } \\
\text { cama? }\end{array}$ & 1,82 & 0,18 \\
\hline $\begin{array}{l}\text { F-6. O seu problema restringe muito a sua } \\
\text { participação em atividades sociais, como sair } \\
\text { para jantar, ir ao cinema, dançar ou ir às festas? }\end{array}$ & 1,45 & 0,00 \\
\hline $\begin{array}{l}\text { F-7. Por causa do seu probl ema, você tem } \\
\text { dificuldade para ler? }\end{array}$ & 0,73 & 0,55 \\
\hline $\begin{array}{l}\text { F-8. A execução de atividades como esportes, } \\
\text { dança, pequenas tarefas caseiras, como varrer ou } \\
\text { retirar os pratos, aumenta seu problema? }\end{array}$ & 2,00 & 1,45 \\
\hline $\begin{array}{l}\text { E-9. Você tem medo de sair de casa sem que } \\
\text { alguém o(a) acompanhe por causa do seu } \\
\text { problema? }\end{array}$ & 1,27 & 0,36 \\
\hline $\begin{array}{l}\text { E-10. Você se sente envergonhado(a) diante de } \\
\text { outras pessoas por causa do seu problema? }\end{array}$ & 0,91 & 1,27 \\
\hline $\begin{array}{l}\text { O-11. Movimentos rápidos de cabeça aumentam } \\
\text { seu problema? }\end{array}$ & 2,55 & 1,82 \\
\hline $\begin{array}{l}\text { F-12. Você evita alturas por causa de seu } \\
\text { problema? }\end{array}$ & 3,09 & 1,45 \\
\hline O-13. Virar-se na cama aumenta seu problema? & 1,09 & 0,73 \\
\hline $\begin{array}{l}\text { F-14. È difícil para você realizar trabalhos } \\
\text { caseiros? }\end{array}$ & 1,27 & 0,91 \\
\hline $\begin{array}{l}\text { E-15. Em decorrência do seu problema, você } \\
\text { tem medo que as pessoas pensem que está } \\
\text { drogado(a)? }\end{array}$ & 1,45 & 0,91 \\
\hline $\begin{array}{l}\text { O-16. È difícil de caminhar sozinho(a) por causa } \\
\text { do seu problema? }\end{array}$ & 1,09 & 0,18 \\
\hline $\begin{array}{l}\text { O-17. Andar numa calçada aumenta seu } \\
\text { problema? }\end{array}$ & 0,91 & 1,45 \\
\hline $\begin{array}{l}\text { E- } 18 \text {. Você sente dificuldade para se concentrar } \\
\text { por causa do seu problema? }\end{array}$ & 2,18 & 1,09 \\
\hline $\begin{array}{l}\text { O-19. Em razão do seu problema, é difícil para } \\
\text { você andar pela casa no escuro? }\end{array}$ & 1,45 & 1,45 \\
\hline $\begin{array}{l}\text { E-20. Por causa do seu problema, você tem } \\
\text { medo de ficar sozinho(a) em casa? }\end{array}$ & 1,27 & 0,36 \\
\hline $\begin{array}{l}\text { E- } 21 \text {. Você se sente prejudicado(a) em razão do } \\
\text { seu problema? }\end{array}$ & 3,27 & 1,64 \\
\hline $\begin{array}{l}\text { E-22. O seu problema provocou tensão em seu } \\
\text { relacionamento com familiares ou amigos? }\end{array}$ & 0,91 & 0,18 \\
\hline $\begin{array}{l}\text { E- } 23 \text {. Você se sente deprimido(a) por causa do } \\
\text { seu problema? }\end{array}$ & 1,45 & 0,91 \\
\hline $\begin{array}{l}\text { F-24. O seu problema interfere no trabalho ou } \\
\text { em suas responsabilidades com afazeres } \\
\text { domésticos? }\end{array}$ & 1,64 & 1,09 \\
\hline O-25. Abaixar a cabeça aumenta seu problema? & 3,27 & 2,18 \\
\hline
\end{tabular}


Tabela 3 - Média das escalas emocional, orgânico-física e funcional do DHI pré e pós-reabilitação. Baurú, SP, 2010.

\begin{tabular}{lccc}
\hline Média das escalas & Pré-reabilitação & Pós- reabilitação & Comparação \\
\hline Emocional & 1,71 & 0,90 & 0,81 \\
Orgânico-física & 1,89 & 1,35 & 0,54 \\
Funcional & 1,71 & 0,82 & 0,89 \\
\hline
\end{tabular}

antecedentes mórbidos de cada paciente podem revelar alguma doença pessoal ou familiar que possa estar implicada na origem das tonturas, tais como afecções hereditárias, distúrbios metabólicos e/ou cardiovasculares, alterações visuais, migrânea, diabetes mellitus, esclerose múltipla e depressão, dentre outras doenças associadas. ${ }^{27,28}$

A prevalência da configuração audiométrica encontrada foi de acordo com o esperado para a faixa etária estudada, ou seja, configurações características da presbiacusia, isto é, perda auditiva bilateral, progressiva, com rebaixamento nas frequências mais altas. Prejudicou-se assim a inteligibilidade de fala, acompanhada ou não de zumbido, ${ }^{18}$ podendo ser causada pelo aumento da rigidez das membranas tectória e basilar e de outras partes do órgão de Corti, que tendem a se agravar com o passar da idade. ${ }^{29}$

A história clínica associada aos resultados das avaliações realizadas evidenciou que a maioria dos participantes apresentava transtornos vestibulares periféricos.

Entre as vestibulopatias periféricas, as vertigens posturais caracterizadas como VPPB são comuns em indivíduos da população geriátrica. Muitas vezes, o paciente consegue relatar quais movimentações posturais desencadeiam tais sintomas. ${ }^{12,28}$

A VPPB é considerada uma das vestibulopatias periféricas mais frequentes, prevalente nos idosos e em pacientes do gênero feminino. Uma das principais e mais utilizadas opções terapêuticas para a VPPB consiste em manobras de reposicionamento dos otólitos de volta ao utrículo. Uma das manobras de reposicionamento mais conhecidas é a Manobra de Epley, descrita em 1992, que apresenta excelentes resultados na prática clínica. ${ }^{30}$

Diversos são os fatores que podem interferir no processo terapêutico. $\mathrm{O}$ uso $\mathrm{da}$ multimedicação ou automedicação desnecessária pode ser a causa da tontura vestibular e sintomas associados, além de hábitos inadequados de alimentação, vícios (álcool, fumo, abuso de café), vida sedentária e estresse, que podem ser causas ou importantes fatores agravantes de labirintopatias. O acompanhamento psicológico pode ser necessário em alguns casos, frequentemente associado à ansiedade, depressão, pânico ou insegurança física. ${ }^{28,29}$

A atividade física associa-se a numerosos benefícios à saúde, pois os exercícios, além de promoverem melhora do metabolismo geral, são importantes na estimulação proprioceptiva e postural, promovendo a aceleração da compensação vestibular. Estudo indica que indivíduos com vida social ativa, praticantes de algum tipo de atividade física tendem a apresentar menos queixas de tonturas. ${ }^{5} \mathrm{Uma}$ vida sedentária associada a distúrbios do sistema vestibular pode provocar forte impacto na qualidade de vida dos pacientes. ${ }^{14}$

Estudos relatam que as maiores causas dos problemas metabólicos são as dietas inadequadas e a indisciplina alimentar, que influem em aumento da ingestão de açúcares e gorduras. As refeições devem ser distribuídas de forma adequada ao longo do dia, em três etapas: desjejum, almoço e jantar com pequenos lanches entre elas. ${ }^{31}$

A conduta mais recomendada atualmente refere-se ao esclarecimento diagnóstico do quadro 
labiríntico ao paciente e a conscientização da adesão no processo terapêutico. Os médicos têm optado, cada vez mais, pelo encaminhamento para RV e adequação dos hábitos de vida, sendo os medicamentos utilizados apenas em alguns casos. O prognóstico das labirintopatias periféricas costuma ser excelente, com a terapia otoneurológica por meio da realização das manobras de reposição labiríntica, integrada a RV, tratamento etiológico, orientação nutricional, modificação de hábitos, medicação antivertiginosa e eventual aconselhamento psicológico. ${ }^{32}$

Baseando-se na literatura ${ }^{6,8,9,26,29}$ e nos resultados do presente estudo, a RV mostrou-se uma proposta terapêutica eficiente no tratamento das labirintopatias. A elaboração de uma proposta personalizada favoreceu a diminuição das queixas trazidas pelos pacientes, fazendo com que a abordagem terapêutica fosse mais eficiente, o que corrobora com o referencial teórico. ${ }^{33}$

O questionário de handicap para tontura permitiu verificar o grau de desvantagem que a disfunção vestibular causa na vida do paciente. Neste estudo, a escala física foi a mais afetada, provavelmente pelo fato de muitos indivíduos idosos levarem uma vida sedentária e estarem afastados de atividades profissionais e muitas vezes sociais, fazendo com que pequenas atividades domésticas passem a exigir maior atenção em sua execução.

Fielder et al., em 1996, ${ }^{34}$ também verificaram que tanto para os homens quanto para as mulheres o desempenho das funções físicas foi significativamente mais afetado pelos efeitos da vertigem em relação aos outros aspectos avaliados pelo DHI. Estudo em idosos apontou que o comprometimento das escalas orgânico-física e funcional foi maior que a emocional antes da aplicação dos exercícios. Após a RV, observou-se melhora no aspecto total e nas escalas avaliadas, sendo que a física apresentou melhora significativa em relação às outras. ${ }^{14}$
A RV demonstrou ser um procedimento terapêutico que proporciona melhora sintomatológica em curto período de tratamento para os idosos. No entanto, a necessidade de adesão a todas as condutas e orientações propostas para a RV e a existência de doenças associadas ao quadro labiríntico caracterizam limitações nesse estudo.

Baseando-se nos resultados, sugere-se a aplicação desses procedimentos em outras populações idosas e em outras faixas etárias, o que possibilitaria apresentar programas nos diversos níveis de saúde.

\section{CONCLUSÃO}

Os achados deste estudo possibilitaram concluir que a múltipla abordagem diagnóstica e terapêutica foi um importante recurso no diagnóstico e prognóstico dos transtornos labirínticos dos pacientes idosos participantes, com prevalência de síndrome vestibular periférica irritativa e predomínio de vertigem postural paroxística benigna, a qual apresentou melhor prognóstico.

A RV individualizada, associada às orientações quanto a mudanças de hábitos deletérios, adequação da alimentação e práticas de atividade física regular, contribuiu para o desaparecimento ou diminuição dos sintomas, sendo que para a maioria dos casos de VPPB a manobra de reposicionamento foi o único recurso terapêutico utilizado.

A RV mostrou-se um método fácil de ser aplicado e capaz de proporcionar melhora na qualidade de vida, envolvendo os aspectos biopsicossociais dos pacientes idosos, mesmo quando o desaparecimento dos sintomas não foi total.

O DHI mostrou-se de fácil aplicação e a análise de seus dados pré e pós-tratamento foi fundamental para monitorar e quantificar a evolução terapêutica. 


\section{REFERÊNCIAS}

1. Mitre EL. Aspectos otorrinolaringológicos do idoso. In: Suzuki HS. Conhecimentos essenciais para atender bem o paciente idoso. São José dos Campos: Pulso; 2003. p. 25

2. Ramos LR. Fatores determinantes do envelhecimento saudável em idosos residentes em centro urbano: projeto epidoso. Cad. Saúde Pública 2003;19 (3): 793-98

3. Instituto Brasileiro de Geografia e Estatística( IBGE). Perfil dos idosos responsáveis pelo domicilio. [Acesso em 12 jun 2006]. Disponível em: http://www.ibge.gov.br/home/presidencia/ noticias/25072002pidoso.shtm.

4. Organização Pan-Americana da Saúde. Envelhecimento saudável 2006 . [Acesso em 12 jun 2006]. Disponível em: http:// www.opas.org.br/ mostrant.cfm? codigodest $=197$

5. Ruwer LS, Rossi AG, Simon LF. Equilíbrio no idoso. Rev. Bras. Otorrinolaringol 2005; 71 (3): 298-303

6. Fukuda Y. Distúrbio vestibular no idoso. In: Ganança MM, Vieira RM, Caovilla HH. Princípios de Otoneurologia. São Paulo: Atheneu; 1998 p.69-73

7. Belal JR, Gloric A. Desequilibrium of aging. J. Laryngol Otol 1986;100:1037- 41.

8. Bittar RSM, Simoceli L, Pedalini MEB, Bottino MA. Repercussão das medidas de correção das comorbidades no resultado da reabilitação vestibular de idosos. Rev. Bras. Otorrinolaringol 2007;73 (3): 295-8

9. Ganança FF, Ganança CF. Reabilitação vestibular Princípios e Técnicas. In: Ganaça MM, Munhoz MSL, Caovilla HH, Silva MLG. Estratégias Terapêuticas em Otoneurologia. São Paulo: Atheneu; 2000. v.4, cap 3.

10. Simoceli L, Bittar RMS, Bottino MA, Bento RF. Perfil diagnóstico do idoso portador de desequilíbrio corporal: resultados preliminares. Rev. Bras. Otorrinolaringol 2003; 69(6):772-77

11. Zanardini FH,et al. Reabilitação vestibular em idosos com tontura. Pró-Fono Rev Atualização Cientifica 2007;19 (2):177-84

12. Resende CR, Taguchi CK, AlmeidaJG; Reabilitação vestibular em pacientes idosos portadores de vertigem posicional paroxística benigna. Rev. Bras. Otorrinolaringol 2003;69(4):34-38
13. Taguchi CK. Reabilitação Vestibular. In: Ferreira LP, Befi-Lopes DM, Limongi SCO. Tratado de Fonoaudiologia .São Paulo: Roca; 2004.

14. Silveira SR, Taguchi CK, Ganança FF. Análise comparativa de duas linhas de tratamento para pacientes portadores de disfunção vestibular periférica com idade superior a sessenta anos. Acta AWHO. 2002 jan-fev;21(1).

15. Jacobson GP, Newman CW. The development of the dizziness handicap inventory. Arch Otolaryngol Head Neck Surg 1990; 116: 424-7.

16. Castro ASO. Dizziness handicap inventory: adaptação cultural para o português brasileiro, aplicação e reprodutibilidade e comparação com os resultados a vestibulometria. . São Paulo: Univ. Bandeirante de SP; 2003.

17. Bureau International d'AudioPhologia. Audiometric classification of hearing impairment. [Acesso em 18 aug 2006]. Disponível em: http://www.biap.biapanglais/ rec021.eng.htm.

18. Santos TM, Russo ICP. A Prática da Audiologia Clínica. 5 ed. São Paulo: Editora Cortez; 2005.

19. Herdman SJ, Tusa RJ. The diagnosis and treatment of benign paroxysmal positional vertigo Schaumburg : ICS Medical Corporation; 1999.

20. Caovilla HH, Ganança MM, Munhoz MSL, Silva MLG. Equilibriometria Clínica. São Paulo: Atheneu; 2000.

21. Mangabeira APL. Atlas de Vectoelectronistagmografia. São Paulo: Ache; 1984.

22. Mizukoshi K, Watanabe Y, Shoyaku H, Okubo J, Watanabe I. Epidemiological study on benign paroxysmal positional vertigo. Acta Otolaryngol (Stockh) 1988: 105 (Supl 447);67-72.

23. Pollak L, Davies RA, Luxon LL. Effectiveness of the particle repositioningmaneuver in benign paroxysmal positional vertigo whit and whithout additional vestibular pathology.Otol. Neurotol 2002;23(1):79-83.

24. Koga KA, Resende BD, Mor R. Estudo da prevalência de tonturas/vertigens e das alterações vestibulares relacionadas a mudança de posição de cabeça por meio da vectoeletronistagmografia computadorizada. Rev Cefac 2004; 6(2):197-202 
25. Ganança MM, Caovilla HH. A vertigem e Sintomas Associados. In: Ganança MM, Vieira RM, Caovilla HH. Princípios de Otoneurologia. São Paulo: Atheneu;1998. v. 1.

26. Ganança FF, Castro ASO; Branco FC, NatourJ. Interferência da tontura na qualidade de vida de pacientes com síndrome vestibular periférica. Rev. Bras. Otorrinolaringol 2004;70(1):94-101

27. Ganança MM, Caovilla HH, Munhoz MSL, Silva MLG .Como diagnosticar e Tratar Alterações da Audição e do Equilíbrio Corporal no Idoso. Rev. Otorrinolaringol 1999; 56(10).

28. Gushikem P, Caovilla HH, Ganança MM. Avaliação otoneurológica em idosos com tontura. 2001. [Acesso em 05 jun 2006]. Disponível em: http:// www.actaawho.com.br

29. Ganança MM, Caovilla HH. Como lidar com as tonturas e sintomas associados. In: Ganança MM, Munhoz MSL, Caovilla HH, Silva MLG.

Estratégicas terapêuticas em otoneurologia. São Paulo: Atheneu; 2000. p. 1-20.
30. Ganança CF. O exame otoneurológico e a deficiência auditiva. In: Lopes Filho O. Tratado de Fonoaudiologia. Ribeirão Preto: Tecmed; 2005.

31. Ramos S, Ramos RF. Medidas complementares no tratamento da vertigem. In: Ganaça MM. Vertigem tem cura? São Paulo: Lemos Editorial; 1998.

32. Silva MLG, Munhoz MSL, Ganança MM, Caovilla HH. Quadros clínicos otoneurológicos mais comuns. São Paulo: Ed. Atheneu; 2000. v. 3.

33. Nishiro LK, Ganança CF, Manso A, Campos CAH, Korn GP. Reabilitação vestibular personalizada: levantamento de prontuários dos pacientes atendidos no ambulatório de otoneurologia da ISCMSP. Rev. Bras. Otorrinolaringol 2005;71 (4):440-47.

34. Fielder $\mathrm{H}$, et al. Measurement of health status in patients with vertigo. Clin Otolaryngol 1996;21:124-6. 